(C)2009 IEEE. Personal use of this material is permitted. However, permission to reprint/republish this material for advertising or promotional purposes or for creating new collective works for resale or redistribution to servers or lists, or to reuse any copyrighted component of this work in other works must be obtained from the IEEE. 


\title{
Wireless Multimedia Sensor Network Technology: A Survey
}

\author{
Atif Sharif, Vidyasagar Potdar, Elizabeth Chang \\ Digital Ecosystems and Business Intelligence Institute, \\ Curtin University of Technology, Perth, Western Australia \\ atif.sharif@postgrad.curtin.edu.au, v.potdar@curtin.edu.au, e.chang@,curtin.edu.au
}

\begin{abstract}
Wireless Multimedia Sensor Networks (WMSNs) is comprised of small embedded video motes capable of extracting the surrounding environmental information, locally processing it and then wirelessly transmitting it to parent node or sink. It is comprised of video sensor, digital signal processing unit and digital radio interface. In this paper we have surveyed existing WMSN hardware and communication protocol layer technologies for achieving or fulfilling the objectives of WMSN. We have also listed the various technical challenges posed by this technology while discussing the communication protocol layer technologies. Sensor networking capabilities are urgently required for some of our most important scientific and societal problems like understanding the international carbon budget, monitoring water resources, monitoring vehicle emissions and safeguarding public health. This is a daunting research challenge requiring distributed sensor systems operating in complex environments while providing assurance of reliable and accurate sensing.
\end{abstract}

Keywords: WSN, WMSNs, FPGA, MAC, TCP, QoS, Protocol stack, video sensor.

\section{Overview of Wireless Multimedia Sensor Networks (WMSN)}

In wireless communication networks wireless sensor networks (WSNs) have gained significant importance in the last few years. Currently WSNs are targeting a number of application scenarios ranging from civil and military applications to modern healthcare. WSN are basically comprised of scalar sensors capable of measuring the physical phenomenon like temperature, pressure, light intensity, humidity etc. Today WSNs are used on large scale capable of gathering information from the physical environment, processing it and transmitting the processed information to remote server or location [8]. In either of the above listed applications the bandwidth requirements is not stringent and is delay tolerant.
Now the availability of complementary metal-oxide semiconductor (CMOS) camera and small microphones make possible the development of WMSNs capable of gathering the multimedia information from the surrounding environment. The advent of WMSNs has opened a new vision to existing WSNs there by enhancing its existing capability (due to incorporation of vision sensor and high computational engine) and targeted applications like Intelligent Transportation system (ITS), multimedia surveillance sensor networks etc.

Previous research targets the challenges posed by WSNs like limited node computational and communication power, power source, scalability etc. Now with the development of WMSNs additional challenges are added which must also need to be addressed i.e. application specific QoS constraints, coverage area, innetwork processing, high bandwidth demand, heterogeneous multimedia reliability etc

In this paper we describe the key application areas where multimedia information appeals the existing WSN technology to be replaced by WMSN. We then in Section 3 describe its various characteristics architectures like single tier flat having homogeneous sensors, single tier clustered having heterogeneous sensors and multi-tier heterogeneous. In Section 4 we discuss existing research in the field of WMSNs. Section 5 discusses the technical challenges of WMSN technology followed by the WMSN Communication protocol stack in Section 6 . Finally we have concluded the paper in Section 7.

\section{Applications of WMSNs}

The availability of low cost CMOS camera, audio sensor, low power computational and communication modules has led the WMSNs to target various new applications, which significantly enhance the existing WSNs capability. Some of the key applications are broadly classified into Surveillance, Traffic Monitoring, Personal and Health Care, Habitat Monitoring and Target Tracking. 
WMSNs are currently used in Surveillance applications that require streaming multimedia contents, high bandwidth and advanced signal processing of the gathered data. This enables the law enforcement agencies to cater an unexpected event. The introduction of audio and video sensor to the existing WSNs has enabled the WMSNs node to record activities like thefts, traffic violations/accidents, fire conditions etc. and reports these events in the form of snapshot or streaming video to the server or control station.

Road traffic congestion and its management is now a days a big issue [1] and need to be addressed in order to avoid long traffic jams and more important to save fuel and reduce emissions. The advent of WMSN helps researchers in building a smart ITS solution which will provide a real time traffic status for intelligent traffic routing [1]. It also helps the law enforcement agencies to identify the cause of accident and to identify the violator. WMSNs are also becoming famous for this specific application because of its ease of deployment, low cost and its ease of reconfiguring routes when deployed to a new location.

Another key application targeted by WMSNs is Elderly Personal and Health Care. Now days WMSNs are helping physicians to identify the causes of illness that affect elderly [5]. WMSNs with the incorporation of some telemedicine devices are being used to remotely monitor the patient's body temperature, blood pressure, breathing activity etc. Also with the help of multimedia sensors the patient's serious conditions can be detected and can be contacted to patient's relative (or next to kin) in the time of emergency.

WMSNs are also used in Habitat Monitoring application e.g. sandbar evolution, to census the populations of animals. In this case the deployed sensor network takes time critical multimedia information and conveyed wirelessly to the central control station. The major development in this regard has been taken out by Intel Research Laboratory at Berkeley in collaboration with the College of the Atlantic in Bar Harbor and the University of California at Berkeley who deployed the WMSNs in the Great Duck Island, Maine.

Target Tracking is another application where WMSNs plays its significant role. The target is equipped with some acoustic sensor, whose signals are being received by the sensor node, these signals are processed by the algorithms running on the node to track and forecast the target position. Based on this forecasted target's position the sensor nodes near to the forecasted position are awakened. The sensor's node processing is aimed to perform target localization in distributed manner and to reduce the uncertainty of detection.

\section{WMSN Sensor Network Architecture} In this section we explain the three main network architectures for WMNS. Basically WMSN network architecture as shown in Fig. 3. can be broadly classified into three categories depending on the nature of targeting application.

- Single-tier flat architecture having homogeneous sensors.

- $\quad$ Single-tier clustered architecture having heterogeneous sensors.

- Multi-tier heterogeneous architecture with heterogeneous sensors support.

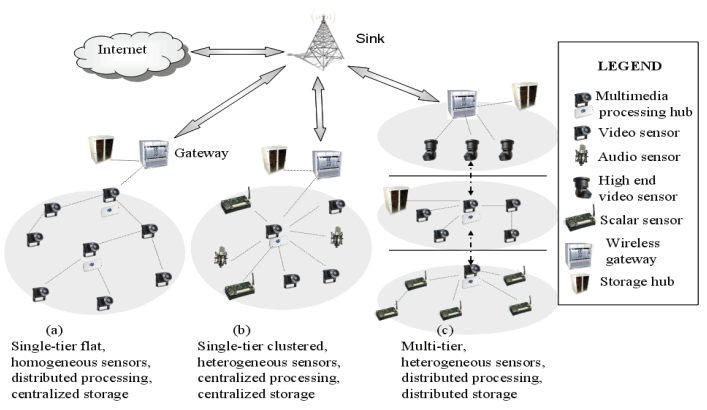

Fig. 3 Architecture of WMSN [8]

The single-tier flat architecture as shown on the left of Fig. 3 composed of homogeneous sensor nodes having the same sensing, computational and communication capability besides the use of same video sensors. The nodes serve two purposes either used for basic multimedia information extraction from surrounding environment or used as multimedia processing hub, which is computationally more powerful than video sensor node. The multimedia information is wirelessly transferred in hop-by-hop fashion from the source nodes to sink/storage device via the gateway. This architecture offers benefits like distributed processing, easy to manage because of homogeneous nature of nodes, nodes are low powered resulting in long network life time.

The middle cloud in Fig. 3 represents the second type of WMSNs architecture, single-tier clustered architecture, composed of heterogeneous sensory nodes (multimedia sensor nodes, basic WSN or scalar sensor nodes etc).

The sensor nodes in the cluster gathers scalar as well as multimedia information and 
sends it to the cluster head which act as central processing unit for that cluster ( having more resources and computational power as compared to other cluster nodes). The processed information is then wirelessly transmitted to sink/storage device via the gateway. The advantage of using this architecture is that it can address a range of application scenarios ranging from simple scalar application to multimedia information processing.

The multi-tier architecture is shown at right in Fig. 3. This architecture is comprised of three tiers. The first tier is composed of scalar WSN nodes for performing simple tasks of gathering the scalar information from the surrounding environment. The middle tier comprised of medium resolution video sensor nodes capable of gathering multimedia information from the surrounding environment. And the final tier composed of high-end vision sensor nodes for complex task like object recognition, tracking objects features etc. Every tier has a central processing hub which is basically a video node having more computational and communication resources. So the storage and the data processing can be performed in the distributed fashion at each different tier. The high end video sensors gather information from the child-tier's video processing hubs in addition to its own gathered information from the targeted location, relayed the processed data wirelessly to the gateway for storage or to the sink. Such a network offers advantages like better scalability, high functionality, reliability and better coverage as compared to single-tier network architecture.

\section{State of the Art}

In this section we discuss the state of the art research in the field of multimedia sensor node development. The initial breakthrough in the CMOS implementation of video sensor node is discussed in [13]. The proposed vision sensor incorporates the System-on-Chip (SoC) smart camera based edge detection.

For real time detection of people and analyzing their movements, the researchers in [11] proposed a smart camera prototype (Hi8 camera for image acquisition) interfaced with standard PC with additional PCI-boards featuring TriMedia TM-1300 VLIWM processor. Another breakthrough is the development of embedded version of vision sensor [7] that uses TMS320C64xx DSP processor and CMOS camera. This camera is equipped with $1 \mathrm{MB}$ onchip memory and $256 \mathrm{MB}$ external memory.
Wired Ethernet connection was being used for communication and configuration.

Another DSP and FPGA based smart camera prototype for multimedia information processing was proposed in [2]. TRICam is equipped with 1 $\mathrm{MB}$ on-chip and $16 \mathrm{MB}$ external memory. In this case the analog video either PAL or NTSC was captured and processed by hardware comprising DSP processor and FPGA.

A further interesting development was the Cyclops as shown in Fig 4.a [9]. Cyclops composed of CMOS Agilent ADCM-1700 CIF camera module, Complex Programmable Logic Device (CPLD) and memory for high speed data communication. It provides an electronic interface between the vision sensor and WSN node like Micaz etc.

CMUcam3 [12], as shown in Fig 4b, is an embedded version of CIF resolution $(352 \times 288)$ RGB color sensor and supports JPEG compression and basic image manipulation library. It grabs image from the color sensor at a rate of 26 frames per second and stores it into onboard memory. CMUcam3 does not support digital radio interface so like Cyclops it needs to be interfaced with some WSN node like TelosB via a serial communication channel [13].

Researchers in [14] developed Cognitive Systems with Interactive Sensors (COGIS) module has ARM7 32 bit CPU (takes short time to process multimedia processing algorithms) clocked at $48 \mathrm{MHz}$ with external storage, ZigBee compliant (802.15.4 standard) Chipcon's CC2420 digital radio interface, Agilent ADCM1670 CMOS vision sensor and 30x30 pixel low resolution CIF optical sensor. The proposed solution showed better results as compared to previous 8-bit architecture supported (Atmel microcontrollers) WSN nodes.

Stargate board [10] as shown in Fig 4c was designed by Intel and produced by Crossbow. When combined with Logitech webcam it can act as a medium resolution WMSNs mote. It has Intel's PXA-255 XScale $400 \mathrm{MHz}$ RISC processor (only for fixed point arithmetic's), 32 Mbyte of Flash memory, 64 Mbyte of SDRAM, and an on-board connector for Crossbow's MICA2 [10] or MICAz [11] motes in addition to PCMCIA Bluetooth or IEEE 802.11 cards. It has high processing capability and more onboard resources as compared to Cyclopes and CMUcam3. This thing led to their use not only as an independent WMSN mote but also as a processing hub for multimedia in-network processing in WMSNs. However it is not good from the energy consumption point of view and 
consumes more energy which is against the design objective of WMSN. Also one has to implement energy efficient floating point operations for multimedia processing.
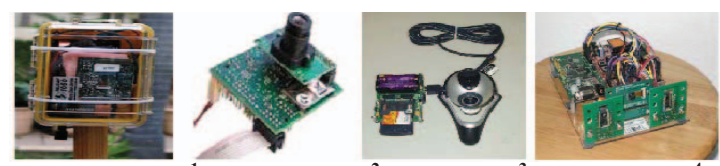

Fig4a. Cyclops ${ }^{1}$ b. CMUcam3 ${ }^{2}$ c. Stargate ${ }^{3}$ d. MeshEye $^{4}$

${ }^{1}$ www.ece.gatech.edu, ${ }^{2}$ www.robot-electronics.co.uk ${ }^{3}$ www.ece.gatech.edu, ${ }^{4}$ wsnl.stanford.edu

Researchers in [3] have presented a smart camera mote architecture targeting the intelligent surveillance application. The MeshEye ${ }^{\mathrm{TM}}$, as shown in Fig 4d, hardware composed of AT91SAM7S family microcontroller, USB 2.0 full speed port ,serial interface, eight kilo pixel imagers, Agilent's CMOS camera ADCM-2700 and Agilent's ADNS-3060 high performance optical mouse sensor. It also incorporates MMC/SD flash memory for temporary buffering. It uses ZigBee/802.15.4 transceiver Chipcon's CC2420 radio chip. The MeshEye ${ }^{\mathrm{TM}}$ is used for real time object detection and in-node processing applications like distributed intelligent surveillance.

A dual camera implementation of WSN node is presented in [4]. The proposed WMSNs network composed of dual camera nodes. The system is two-tiered i.e. low power and high power. The low power tier contains low power sensor nodes comprised of Cyclops camera module, Micaz mote and IGB flash for storage. While the high power tier contains high end processing sensor nodes (Intel's Imote2), high fidelity Enalab camera module and 1GB SD card for image storage. The field of view of both cameras is same because of being closely mounted. It supports Zigbee/802.4.15 radio standard and can be queried from a PC or PDA that is connected to 802.4 .15 radio. The system uses low power tier for still object detection and high power tier is being used for energy efficient accurate object recognition/classification.

There has been a lot of work undertaken in this field however the technology is still not mature enough and several technical challenges need to be addressed, which are application specific and need special consideration. The next section outlines these challenges.

\section{Technical Challenges}

In this section we will discusses the technical challenges subject to WMSNs. Since in WMSNs most of the communication is multimedia by nature i.e. either snapshot of an event or streaming contents, so a number of factors would influence the design of video sensor network among them are QoS Requirement, Scalable and flexible architectures and protocols to support heterogeneous applications, High bandwidth demand, Localized processing and data fusion, Power efficient design, Reliability and Integration with IP and various other wireless technologies.

\subsection{QoS requirement}

One of the first and the most important challenge to the WMSNs design is to meet the application specific QoS requirements. The WMSNs are designed to address a range of application scenarios ranging from simple scalar application to multi-tier support involving heterogeneous sensors that includes multimedia sensor support besides the use of scalar sensors. In former case bandwidth is not a big issue and is easily handled, confirming to application specific QoS requirements. While in later case the inclusion of multimedia information i.e. snapshot (event based) or streaming media (long duration possibly) may impose strict constraints on QoS requirements besides the high bandwidth requirement. This requires the strong coordination between the application specific algorithms/protocols of communication protocol layers (application, transport, network, MAC/data link and Physical layer) and supporting hardware in order to meet the application specific QoS requirements.

\subsection{Scalable and flexible architectures and protocols to support heterogeneous Applications}

WMSNs design should be scalable and flexible enough for future network expansion. The multimedia processing algorithms and communication layer protocol are flexible enough to support a range of multimedia applications while ensuring the application specific QoS requirements, energy, delay and privacy constraints of WMSNs.

\subsection{High bandwidth demand}

In WMSNs the bandwidth requirement for multimedia data communication is order of magnitude higher than the bandwidth required for the existing WSNs. For example the scalar WSN architecture involving motes like TelosB or Micaz etc support Zigbee/802.15.4 radio standard that supports the data rate of up to $250 \mathrm{Kbps}$. However in WMSNs this bandwidth 
may not full fill the purpose so Zigbee/82.15.4 radio standard may not be suitable for multimedia information communication rather we can think of UWB or Impulse radio technologies (Physical layer) for meeting the WMSN bandwidth requirements.

\subsection{Localized processing and data fusion}

In WMSNs communicating multimedia information requires large bandwidth and also the communication cost would be enormous if we communicate the information unprocessed. This thing arose the need of in network processing. The WMSN node has high computational capability so the effective use of application layer multimedia processing and data fusion algorithms may not only helps in reducing the high bandwidth demand but also lowers the communication cost. So instead of using the traditional predictive encoders $[6,8]$ that are more complex and energy consuming, the idea of distributed coding that employs simple encoders seems promising for energy constrained WMSN.

\subsection{Power efficient design}

WMSNs are considered to have strict power constrains as compared to traditional WSNs. The multimedia information processing algorithms and its communication at high rate are the most energy consuming processes in WMSNs. This requires development of energy aware multimedia processing algorithms (application layer algorithms) and communication protocol stack/routing [8, 20] both the transport and network layer protocols) in order to maximize the network life time while meeting the application specific QoS constraints.

\subsection{Reliability}

In WMSN the reliability of node as well as data transfer is of great concern. In most cases the power depletion is the only cause of node's failure other may include harsh hostile environment or physical damage. Another major cause of node's power depletion is the extensive packet retransmissions due to unreliable data transfer by the transport functions. So this thing reveals that the design of WMSNs should be reliable enough to withstand against such unwanted occurrences and this can be achieved by making node's physical structure robust and use of reliable, power efficient transport functions.

\subsection{Integration with IP and various other wireless technologies}

The WMSNs design should support various other wireless communication standards like Bluetooth, Wi-Fi etc and Internet Protocol (IP) suite. This may enables the user to pop the network's information from anywhere any time. Also with the support of various wireless technologies it would be possible to interact with other wireless networks without sacrificing the operational efficiencies within each individual technology [8]. This communication interface with other nodes or other wireless technologies is supported by the physical layer of the WMSN communication protocol stack which will be discussed in the next section.

\section{Communication Protocol Stack}

This section talks about the communication protocol stack of WMSNs. It also describes the key challenges and the related work specific to the communication layer. The communication protocol stack of WMSNs comprised of application layer, transport layer, network layer, MAC layer and physical layer. For an efficient design of WMSNs it is important that all the layers of the communication protocol stack should support functions which are resource and power efficient.

\subsection{Application layer}

The application layer in WMSNs supports multimedia coding techniques which are in accordance to the application specific requirements and hardware posed constraints. Since the information is multimedia by nature so the source coders should be less complex and have high compression efficiency without degrading the application QoS. Less complexity leads to power efficient design of the source coders. Another key feature of such coders is that they communicate reliably over lossy links. Various techniques like Intra-frame or interframe also called predictive coding or motion estimation techniques are complex in nature (not power efficient) [8]. Another proposal [17] is to shift the complexity from node's to sink end, this method is a part of distributed source coding i.e. Wyner-Ziv coding technique which gives results comparable to predictive motion estimation technique which employs complex encoders at the node's end.

In addition to support the source coding techniques the application layer also supports network traffic management and admission control functionalities which is directly related to application specific QoS. So based on class of traffic the application layer offers differentiated 
services in order to meet the application specific QoS [18].

\subsection{Transport layer}

In WMSNs the transport layer is responsible for transporting the source node's data collected from the surrounding environment to sink. As in WMSNs the majority of the applications are event critical so the reliable data transfer is the main objective of WMSNs transport protocol design in addition to support high data rate/congestion control $[19,20]$ features of application. In WMSN when the multimedia information is streaming by nature then it is critically important that the sink receives data in ordered sequence in which source sends data otherwise the information become redundant this stress the need of transport's layer packet level reordering[8].

Since the information is multimedia by nature so it requires high data rate support and there always a chance of congestion occurrence at any point of the network especially at the sink node, result would be in depletion of node's energy. So a need arises for power efficient congestion control algorithms in order to avoid data oscillations.

TCP, a connection oriented approach, provides strict end-to-end reliability which is a good feature from the reliability prospective but not good from the energy efficiency point of view because TCP demands packet acknowledgements for every data packet transmission and retransmission of data packet in case of failure besides the overhead caused by the TCP link establishment. UDP (power efficient than TCP) on the other hand did not follow the strict reliability paradigm like TCP but lacks reliability. Existing WSN transport layer protocols like Congestion Detection and Avoidance CODA [20], Multi-flow Real-time Transport Protocol (MRTP) [21], RMST [25], PSFQ [19], GARUDA [23] and STCP [26] etc are designed for upstream or downstream reliability in WSNs are either sink-to-sensor [29, $30]$ or sensor-to-sink reliable delivery [22, 24, and 25]. But none of them supports the real time communication as demanded in WMSNs.

\subsection{Network layer}

Similar to computer networks, in WMSNs the Network layer of the communication protocol stack supports routing functions/protocols that deliver the sensed information from the source node to sink. Here in WMSNs the role of network layer supporting functions/protocols is quite critical as to conserve energy since node has limited energy budget. The multimedia nature of the data (specially the streaming nature) imposes strict constraints to these routing functions/protocols design in order to meet the tight constraints of application specific QoS and reliability requirements. These routing protocols are categorized into three main classes: traffic class based (delay tolerant/intolerant, loss tolerant/intolerant), network condition based and real time streaming based.

A multi path routing protocol [28] was designed to meet the QoS and energy constraints of application. However for real time traffic it does not prioritized the traffic having different QoS requirements. Moreover the protocol is not scalable by nature because during path computation the node should have the entire network topology. The prioritized data routing subject to the constraints like latency tolerance and hand-off dropping rate is proposed in [12] for using mobile sensor networks in telemedicine applications.

Another protocol named SPEED [30] regards delay as well as congestion; it uses a technique called back-pressure re-routing to mitigate congestion. Although SPEED avoids congestion by controlling the data rate but it does not support the prioritization. Another advancement is the Traffic and Energy Aware IEEE 802.15.4 (TEA-15.4) [27] for supporting the multimedia services in WSNs. The behavior of the protocol is adaptive by nature depending upon the changing traffic conditions.

\subsection{MAC/DATA Link layer}

As opposed to WSNs in WMSNs the varying nature of the network traffic can be classified into a number of service classes that requires different buffering, transmission and scheduling policies. MAC/DATA Link Layer protocols in WMSNs are responsible for providing energy efficient, reliable and error free communication to a range of traffic classes while supporting the application specific QoS requirements. Channel access policies, scheduling and buffer management and link error control are the key areas subject to this layer where the recent research is being going on. Also packet collision, retransmission, over-hearing are the key causes of node's energy depletion and are currently being researched at MAC level protocol development that will handle all these issues.

S-MAC [29] (contention based approach) conserves node's energy by toggling it on and 
off state during its life. This protocol is not suitable for multimedia application as it conserves energy at the cost of throughput reduction and latency etc. Also it fails to handle the node synchronization and coordination with neighboring nodes in case of node's dynamic duty cycle and needs to be researched. Similarly T-MAC [16] is designed to handle receiver's buffer overflow during the burst transfer of the multimedia information.

Adaptive MAC protocol was designed with an aim to reduce the end-to-end latency. In this case each node duty cycle is adaptive by nature in accordance to the varying traffic load. This enables for a node to live for its pre configured lifetime. The Adaptive MAC offers less delay as compared S-MAC while meeting the network lifetime requirements.

Clustered on-demand multi channel MAC protocol (COM-MAQ) [15] targets energy efficiency, high throughput and data reliability parameter for multimedia data communication. Packet overhearing, idle listening and collision can be avoided in this protocol by letting the cluster nodes to operate in the contention free mode. In order to maximize the network throughput while simultaneously enhancing the transmission reliability it incorporates trafficadaptive QoS-aware scheduling algorithm and spectrum-aware ARQ.

\subsection{Physical layer}

Physical layer in WMSNs is responsible for providing error controlled physical datacommunication interface with which node interacts with other network nodes. Bluetooth (IEEE 802.15.1), ultra-wideband (UWB, IEEE 802.15.3), Zigbee (IEEE 802.15.4), and Wi-Fi (IEEE 802.11) are four protocol standards for short range wireless communications with low power consumption. In WSNs Zigbee is considered to be the most suitable radio standard that supports data rate of up to $250 \mathrm{kbps}$, coding efficiency of $76.52 \%$, supports more than 65000 nodes and effective within the range of 10-100 meters. Bluetooth and Wi-Fi on the other hand although have high coding efficiencies i.e. $94.41 \%$ and $97.18 \%$ and supports maximum data rate of up to $0.72 \mathrm{Mbps}$ and $54 \mathrm{Mbps}$ respectively but they are not ideally power efficient. On the other hand Zigbee is not suitable to support the high data rate multimedia application in terms of power consumption and application specific QoS.

Ultra wideband (UWB), with coding efficiency of $97.94 \%$ maximum data rate support of
$110 \mathrm{Mbps}$, power efficient than any other wireless technology and nominal range of 10 meters, is considered to be the most ideal candidate for WMSNs. Additionally UWB signals have extremely low-power spectral density, with low probability of intercept/detection. There are two variants of UWB i.e. Time-Hopping Impulse Radio UWB (TH-IR UWB) [8] and Multi-Carrier UWB (MCUWB). TH-IR UWB, considered to be most suitable for WMSNs, enables high data rate, high processing gain in interference, very low-power wireless communications, simple-design, carrier less baseband communications. However recent research shows that high power efficiency while meeting the strict multimedia application QoS constraints can only be achieved by developing cross-layer model for the physical and MAC layers for WMSNs transceiver.

\section{Conclusion}

In this paper we surveyed the WMSNs technology. We have also discussed the existing hardware and the communication protocol stack design. This research survey also figure out a number of technical challenges that this technology imposes on hardware and communication protocol development part of the design. This may leads to the future research direction. Based on this research survey we will next try to make a conceptual frame work for our own WMSNs node which will covers the node hardware and the reliable transport function development aspects of the framework.

\section{References}

[1] Kansas City Scout Intelligent Transportation System, MO, USA, http://www.roadtraffictechnology.com/projects/kansas/, $20^{\text {th }}$ Oct, 2008

[2] C. Arth, H. Bischof, and C. Leistner, "TRICam - An Embedded Platform for Remote Traffic Surveillance," in Proc. of Conf on Comp Vision and Pattern Recognition CVPR Workshop on Emb Sys, 2006, pp. 125-125.

[3] S. Hengstler, D. Prashanth, S. Fong, and H. Aghajan, "Mesheye: a hybrid-resolution smart camera mote for applications in distributed intelligent surveillance," in Proc of $6^{\text {th }}$ Int Conf on Inf Proc in Sensor Net,'07, USA, pp.360-369. [4] X. Dan, Y. Tingxin, G. Deepak, and H. Allen, "Design and Implementation of a DualCamera Wireless Sensor Network for Object Retrieval," in Proc of 7th int conf on Inf proc in sensor net: 2008, USA, pp. 469-480.

[5] F. Hu and S. Kumar, B "Multimedia query with QoS considerations for wireless sensor 
networks in telemedicine", in Proc. Soc. PhotoOptical Instrum. Eng. Int. Conf. Internet Multimedia Manage. Syst., USA, Sep. 2003.

[6] Girod, B., Aaron, A., Rane, S., \& Monedero, D. "Distributed video coding". Proceedings of the IEEE, (2005). 93(1), 71-83.

[7] M. Bramberger, J. Brunner, B. Rinner, and H. Schwabach, "Real-time Video Analysis on an Embedded Smart Camera for Traffic Surveillance," in Proc. of 10th IEEE Real-Time and Emb Tech and App Symp 04, pp.174-181 [8] I.F. Akyildiz, T. Melodia, and K.R. Chowdhury, "A Survey on wireless multimedia sensor networks," Computer Networks (Elsevier) J., vol. 51, pp. 921-960, 2007.

[9] M. Rahimi, R. Baer, O. Iroezi, J. Garcia, J. Warrior, D. Estrin, M. Srivastava, "Cyclops: in situ image sensing and interpretation in wireless sensor networks," in: Proc. of the ACM Conf. on Embedded Networked Sensor Sys, USA, 2005.

[10] Crossbow Mote Specifications. $<$ http://www.xbow.com>. $21^{\text {th }}$ Aug, 08 .

[11] W. Wolf, B. Ozer, and T. Lv, "Smart Cameras as Embedded Systems," IEEE Computer, vol. 35, no. 9, pp. 48-53, Sept. 2002.

[12] Hu, F.; Kumar, S. Multimedia query with QoS considerations for wireless sensor networks in telemedicine. In Proceedings of Society of Photo-Optical Instrumentation Engineers International Conference on Internet Multimedia Management Systems, USA, September 2003.

[13] T. W. J. Moorhead and T. D. Binnie, "Smart CMOS Camera for Machine Vision Applications," in Proc. Of the IEE Conference on Image Processing and its Applications, UK, July 1999 , pp. 865-869.

[14] I. Downes, L.B. Rad, H. Aghajan, Development of a mote for wireless image sensor networks, in: Proc. of Cognitive systems with Interactive Sensors, France, 2006.

[15] Cheng Li; Pu Wang; Hsiao-Hwa Chen; Guizani, M., "A Cluster Based On-demand Multi-Channel MAC Protocol for Wireless Multimedia Sensor Networks Communications," 2008. ICC apos;08. IEEE International Conference on Vol, Issue, 19-23 May 2008 pp. $2371-2376$

[16] T.V. Dam, K. Langendoen, An adaptive energy-efficient MAC protocol for wireless sensor networks, in: Proc. of the ACM Conf. on Embedded Networked Sensor Systems (SenSys), Los Angeles, CA, USA, November 2003.

[17] Z. Xiong, A.D. Liveris, S. Cheng, "Distributed source coding for sensor networks," IEEE Signal Process. Mag. 21 2004, pp. 80-94.
[18] M. Perillo, W. Heinzelman, "Sensor management policies to provide application QoS, Ad Hoc Networks,"(Elsevier) (2-3),03,235-246. [19] C. Y. Wan, A. T. Campbell, and L. Krishnamurthy, "PSFQ: a reliable transport protocol for wireless sensor networks," in Proc. ACM WSNA 2002, pp. 1-11, Sept. 2002.

[20] D. Ganesan, D. Estrin, A. Woo, D. Culler, B. Krishnamachari, and S.Wicker. Complex behavior at scale: An experimental study of lowpower wireless sensor networks. In Technical Report CS TR 02-0013, UCLA, 2002.

[21] Michael R. Garey and David S. Johnson. Computers and Intractability; A Guide to the Theory of NP-Completeness. W. H. Freeman \& Co., New York, NY, USA, 1990.

[22] Tian He, John A Stankovic, Chenyang Lu, and Tarek Abdelzaher. SPEED: A Stateless Protocol for Real-Time Communication in Sensor Networks. In Proc. of ICDCS, Providence, Rhode Island, USA, May 2003.

[23] S. Park, R. Vedantham, R. Sivakumar, and I.F. Akyildiz. A Scalable Approach for Reliable Downstream Data Delivery in Wireless Sensor Networks. In Proc. of ACM MobiHoc, pages 7889, Roppongi Hills, Tokyo, Japan, May 2004.

[24] Y. Sankarasubramaniam, O.B. Akan, and I.F. Akyildiz. ESRT: Event-to-Sink Reliable Transport in Wireless Sensor Networks. In Proc. of ACM MobiHoc, pages 177-188, USA, 2003.

[25] F. Stann, J. Heidemann, RMST: Reliable data transport in sensor networks, in: Proc. of IEEE Sensor Net Protocols and App (SNPA), Anchorage, Alaska, USA, 2003, pp. 102-112.

[26] Y.G. Iyer, S. Gandham, S. Venkatesan, STCP: a Generic Transport Layer Protocol for Wireless Sensor Networks, in: Proc. of IEEE Intl. Conf. on Computer Communications and Networks (ICCCN), USA, 2005, pp. 449-454.

[27] Suh, C.; Mir, Z.H.; Ko, Y.B. Design and Implementation of Enhanced IEEE 802.15.4 for Supporting Multimedia Service in Wireless Sensor Networks. Elsevier Comp Net, 2008.

[28] Akkaya, K.; Younis, M. An energy-aware QoS routing protocol for wireless sensor networks. In Proceedings of Int Conf on Distributed Comp Sys Workshops, 2003.

[29] W. Ye, J. Heidemann, D. Estrin, Medium access control with coordinated, adaptive sleeping for wireless sensor networks, IEEE Trans. Network. 12 (3) (2004) 493-506.

[30] Felemban, E.; Lee, C.G.; Ekici, E. MMSPEED: Multipath multi-SPEED protocol for QoS guarantee of reliability and timeliness in wireless sensor networks, IEEE Trans. Mobile Comput.2006, 5(6), 738-754. 